\title{
Evidence for diet-induced thermogenesis in hyperphagic cafeteria-fed rats
}

\section{By Michael J. Stock and Nancy J. Rothwell, Department of Physiology,} St. George's Hospital Medical School, Tooting, London $S W_{1} 7$

Diet-induced thermogenesis (DIT) is the increase in heat production which follows the ingestion of food or alterations in the plane of nutrition, and therefore includes the energy costs of feeding, digestion and absorption, tissue synthesis and adaptive non-conservative mechanisms. Theoretical and empirically-derived values exist for the energy cost of processing nutrients and tissue synthesis, but the existence and importance of adaptive changes in heat production are more controversial and estimates of the magnitude of this component vary considerably.

Evidence that DIT is important in energy balance regulation has been obtained from a number of experiments involving chronic overfeeding of human subjects (for review see Garrow, I980) and animals maintained on low-protein diets (Miller \& Payne, I962; Lotfi, 1977; Gurr et al. 1980). However, detailed and accurate long-term energy balance studies are difficult to perform in man, and many of the animal experiments could be criticised because of the unphysiological and sometimes bizarre techniques employed to induce hyperphagia. This problem has now largely been overcome by the observation that the rat can be induced to overeat simply by presenting it with a choice of highly palatable food items, a dietary technique known as the 'cafeteria' feeding system (Rothwell \& Stock, 1 979a). Rats presented with the cafeteria diet overeat by up to $80 \%$ compared to controls maintained on a pelleted stock diet, but the effect of this hyperphagia on energy balance and body-weight depends on a number of factors such as age, strain and environmental temperature.

Young (weanling) cafeteria-fed rats overeat by about $80 \%$ but also show large $(70-90 \%)$ increases in energy expenditure and therefore gain little or no excess weight and fat (Rothwell \& Stock, I $980 a$ ). Older (6-8 week) animals gain slightly more weight than controls (Rothwell \& Stock, 1979b) whereas 6-month-old rats gain weight rapidly (Rothwell \& Stock, unpublished results). However, even in the latter group, expenditure was increased by $57 \%$, and therefore restricted the development of obesity. The levels of hyperphagia and thermogenesis are also dependent on environmental temperature and are much greater in cold environments (Rothwell \& Stock, I $980 \mathrm{~b}$ ). It is therefore possible that attempts to induce large changes in energy intake and DIT in thermoneutral environments would be unsuccessful due to inhibitory thermoregulatory influences.

In earlier studies on the energy balance of cafeteria-fed rats we have relied on determinations of metabolizable energy $(\mathrm{ME})$ intake from food composition tables, body energy gain from body composition and energy expenditure by difference (i.e. the comparative carcass technique). The errors associated with these methods are 
very much smaller $\left(<_{10} \%\right)$ than the increases in energy expenditure $(40-90 \%)$ produced by the cafeteria diet. However, in order to dispel any doubts that might exist concerning the importance of DIT, we have recently measured energy intake, expenditure and carcass energy gain, each by two separate methods.

Male, lean Zucker rats of two ages (5 weeks and 6 months) were maintained on a cafeteria diet for $24 \mathrm{~d}$ and $M E$ intake was determined from (a) food tables and (b) from the heat of combustion of each food presented and all spilt food, faeces and urine. Cafeteria-fed rats of both ages consumed more energy than controls and intakes derived by bomb calorimetry (young control $208 \pm 10$, cafeteria $360 \pm 20$; old control $245 \pm 15$, cafeteria $423 \pm 15 \mathrm{~kJ} /$ rat per d) differed by less than $3 \%$ from values obtained from food tables (young cafeteria $353 \pm 20$, old cafeteria $433 \pm \mathrm{r} 5 \mathrm{~kJ} /$ rat per d). Similar agreement was found between the body energy content calculated from fat and fat free mass, and from bomb calorimetry of the carcass. Energy expenditure was calculated from ME intake and body energy gain, and found to be elevated by 77 and $57 \%$ in young and old cafeteria-fed rats respectively, compared to their age-matched controls.

In a separate experiment, energy expenditure was measured in 5-week-old Sprague-Dawley rats, fed on either a stock or cafeteria diet, using both the carcass balance method and indirect calorimetry. The procedure for measuring $24 \mathrm{~h}$ oxygen consumption tended to limit the hyperphagia of the cafeteria group slightly but these animals still consumed 50\% more energy than stock fed controls. Expenditure, determined from energy balance (control $212 \pm 6$, cafeteria $307 \pm 7 \mathrm{~kJ} /$ rat per d; $P<0.00 \mathrm{I})$, was almost identical to that obtained by indirect calorimetry for both groups (control $2 \mathrm{I} 2 \pm 3$, cafeteria $298 \pm 4 \mathrm{~kJ} /$ rat per d).

The results of these two studies confirm the importance of DIT in energy balance regulation, and clearly demonstrate the validity of the methods previously used to estimate energy expenditure in cafeteria fed rats. In every balance experiment undertaken, the energy cost of fat synthesis has been calculated (using a value of $14 \mathrm{~kJ} / \mathrm{g}$, Pullar \& Webster, 1977) and found to account for less than $15 \%$ of the excess energy expenditure of cafeteria-fed rats. However, even this probably overestimates the energy cost of fat synthesis because the cafeteria diet has a high fat content ( $50 \%$ of energy) and most of the lipid deposited probably originated directly from the diet. This is confirmed by the observation that de novo lipogenesis in vivo is markedly depressed in cafeteria rats (Rothwell, Stock and Trayhurn, unpublished results). It is worth noting that empirically determined values for the energy cost of lipid synthesis ( $14 \mathrm{~kJ} / \mathrm{g}$ ) are much higher than theoretical estimates based on ATP requirements (approximately $9.4 \mathrm{~kJ} / \mathrm{g}$ ), which suggests that the former include an element of energetically-wasteful substrate recycling, as well as a small component associated with feeding and absorption.

Other workers have now confirmed the low energetic efficiency and high metabolic rates of hyperphagic cafeteria-fed rats (Tulp et al. 1980; Andrews \& Donne, 198I) but one group (Armitage et al. I98 $\mathrm{I} a, b$ ) have failed to observe any adaptive thermogenesis in these animals. These workers achieved only small increases in energy intake in cafeteria-fed rats $(24 \%)$, and ascribed all of the 
increased expenditure to the energy cost of feeding and lipid synthesis. Armitage et al. (1981 $a$ ) have claimed that the energy expenditure of cafeteria-fed rats cannot be accurately determined from energy intake and body energy gain, and found that this method did not agree with indirect calorimetry. However, there must be some doubt over the validity of the latter measurements. For example, they reported that control rats consumed $\mathrm{I} 60 \mathrm{~kJ} / \mathrm{d}$, but, after correction for a systematic error, this reduces to $155 \mathrm{~kJ} / \mathrm{d}$. Expenditure (determined by indirect calorimetry) was also I $55 \mathrm{~kJ} / \mathrm{d}$, indicating that the animals were in energy balance. The rats in these experiments were only 6-weeks-old, when energy retention should be at least $20-40 \mathrm{~kJ} / \mathrm{d}$. One can only assume that animals were ill, or their diet was inadequate to support growth, or the energy balance measurements were inaccurate.

Apart from possible methodological problems, these studies demonstrate some of the experimental problems that can arise. Any study of overnutrition must obviously include healthy control animals that grow normally, in order to avoid confusing the effects of overnutrition with nutritional rehabilitation. It also follows that in order to demonstrate adaptive thermogenesis, the degree of hyperphagia has to exceed the animal's maximum requirement for body energy gain. Increasing energy intake by only $24 \%$ above maintenance levels in young animals is not sufficient and cannot be compared to the levels (up to 3.6 times maintenance, $1500 \mathrm{~kJ} / \mathrm{W}^{0.75}$ per d) that can be achieved with the cafeteria diet. Finally, it is worth noting that the energy expenditure of cafeteria-fed rats returned to stock diet (Armitage et al. $198 \mathrm{I} b$ ) appeared to be greater than control values, even though the ex-cafeteria-fed rats were hypophagic and losing body energy. This confirms an early finding of ours (Rothwell \& Stock, 1979a) and indicates a contribution from adaptive DIT that cannot be due to a greater nutrient intake or the cost of body energy gain.

\section{REFERENCES}

Andrews, J. F. \& Donne, B. (198I). Proc. Nutr. Soc. 4I, 36A.

Armitage, G., Hervey, G. R., Rolls, B. J., Rowe, E. A. \& Tobin, G. (1981a). F. Physiol., Lond. $3^{16}, 48 \mathrm{P}$.

Armitage, G., Hervey, G. R., Rolls, B. J., Rowe, E. A. \& Tobin, G. (1981b). F. Physiol., Lond. 317, $48 \mathrm{P}$.

Garrow, J. S. (1980). In Recent Advances in Obesity Research II, p. 200 [G. A. Bray, editor]. London: Libbey.

Gurr, M. J., Mawson, R., Rothwell, N. J. \& Stock, M. J. (1980). F. Nutr. I10, 532.

Lotfi, M. (I977). PhD Thesis, University of London.

Miller, D. S. \& Payne, P. R. (1962). 7. Nutr. 78, 255.

Pullar, J. D. \& Webster, J. A. F. (1977). Br. F. Nutr. 37, 355.

Rothwell, N. J. \& Stock, M. J. (1979a). F. Comp. Physiol. Psychol. 93, 1024.

Rothwell, N. J. \& Stock, M. J. (1979b). Nature, Lond. 281, 3 I.

Rothwell, N. J. \& Stock, M. J. (Ig80a). Proc. Nutr. Soc. 39, 5 A.

Rothwell, N. J. \& Stock, M. J. (1980b). Can. F. Physiol. Pharmacol. 58, 842.

Tulp, O., Frink, R., Sims, E. A. H. \& Danforth, E. (1980). Clin. Res. 28, 621 A. 